\title{
Controllability of Nonlinear Impulsive Stochastic Evolution Systems Driven by Fractional Brownian Motion
}

\author{
Juxia Xiong, ${ }^{1,2}$ Guiqing Liu, ${ }^{3}$ and Lijuan $\mathrm{Su}^{2}$ \\ ${ }^{1}$ Chengdu Institute of Computer Application, Chinese Academy of Sciences, Chengdu 610041, China \\ ${ }^{2}$ Guangxi Key Lab of Hybrid Computation and IC Design Analysis, Guangxi University for Nationalities, Nanning 530006, China \\ ${ }^{3}$ College of ASEAN Studies, Guangxi University for Nationalities, Nanning 530006, China
}

Correspondence should be addressed to Juxia Xiong; xiongjuxia1107@163.com

Received 12 June 2015; Accepted 9 July 2015

Academic Editor: Xiaoyu Song

Copyright (C) 2015 Juxia Xiong et al. This is an open access article distributed under the Creative Commons Attribution License, which permits unrestricted use, distribution, and reproduction in any medium, provided the original work is properly cited.

We consider the infinite-dimensional dynamical control systems described by nonlinear impulsive stochastic evolution differential equations. Sufficient conditions for the complete controllability of nonlinear impulsive stochastic systems are formulated and proved under the reasonable assumption that the corresponding linear system is completely controllable.

\section{Introduction}

The impulsive differential systems are valuable tools in the modelling of many processes in which states are changed abruptly at certain moment of time, involving such fields as engineering, physics, and economics, and so forth; see [1-3]. It is well-known that the evolution differential system theory is a generalization of classical theory. So some partial differential systems can be changed into the abstract evolution systems by using semigroup technique. Then the researchers can easily discuss the properties of the partial differential systems by classical differential theory; for more details one can see [4].

The purpose of this paper is to discuss the controllability of the impulsive stochastic evolution systems driven by fractional Brownian motion as the following form:

$$
\begin{aligned}
& d x(t) \\
& \quad=[A(t) x(t)+B(t) u(t)+f(t, x(t), u(t))] d t \\
& \quad+\sigma(t) d B_{Q}^{H}(t), \quad t \in J:=[0, b], t \neq t_{k}, \\
& \begin{array}{ll}
\Delta x\left(t_{k}\right)=I_{k}\left(x\left(t_{k}\right)\right), \quad t=t_{k}, k=1,2, \ldots, p, \\
x(0)=x_{0},
\end{array}
\end{aligned}
$$

where $A(t)$ generates an evolution system $U(t, s)$ on a Hilbert space $X, f: J \times X \times U \rightarrow X, \sigma: J \rightarrow X$. The control function $u(t)$ takes value in $V=L_{2}(J, U)$, and $U$ is a Hilbert space, $B$ is a linear operator from $V$ into $L_{2}(J, X), I_{k}: X \rightarrow X(k=$ $1,2, \ldots, p)$, and $B_{Q}^{H}(t)$ is a $\mathrm{fBm}$ with Hurst index $H \in(1 / 2,1)$ defined in a completely probability space $(\Omega, \Gamma, P)$. Further, $0=t_{0}<t_{1}<\cdots<t_{p}<t_{p+1}=b$ and $x\left(t_{k}^{+}\right)$and $x\left(t_{k}^{-}\right)$ represent the right and the left limits of $x(t)$ at $t=t_{k}$. Also, $\Delta x\left(t_{k}\right)=x\left(t_{k}^{+}\right)-x\left(t_{k}^{-}\right)$represents the jump in the state $x$ at time $t_{k}$ with $I_{k}$ determining, $P C(J, X)=\{x: J \rightarrow X, x$ is continuous for $t \neq t_{k}, x\left(t_{k}^{-}\right)$and $x\left(t_{k}^{+}\right)$exist with $x\left(t_{k}^{-}\right)=$ $\left.x\left(t_{k}\right), k=1,2, \ldots, p\right\}$, and $x_{0}$ is a random variable satisfying $E\left\|x_{0}\right\|^{2}<\infty$.

It is well-known that the noise or perturbations of a stochastic system are typically modeled by a Brownian motion, such as Gauss-Markov. This process has independent increments. However, many researchers have found that the standard Brownian motion is not an effective process in modeling through many physical phenomena. A family of process that seems to have wide physical applicability is fractional Brownian motion (fBm). This process was first introduced by Kolmogorov in 1940. Mandelbrot and Van Ness studied the applications of the $\mathrm{fBm}$ process soon after. Since then various forms of equations have been studied based on different settings. For example, the case of finite-dimensional equations has been studied by Besalú and Rovira [5], Unterberger [6], and Nguyen [7], and the case of infinite-dimensional equations in a Hilbert space has been considered by Boufoussi and Hajji [8], Caraballo et al. [9], and Ahmed [10]. 
One of the basic qualitative behaviors of a dynamical system is controllability, which was first researched by Kalman [11] in 1963. It means that it is possible to steer a dynamical control system from an arbitrary initial state to an arbitrary final state using the set of admissible controls. Many researchers have paid close attention to the study of the controllability for dynamical systems since then. There are many different methods for dealing with the controllability problems for various types of nonlinear stochastic systems. Subalakshmi and Balachandran [12] studied the approximate controllability of nonlinear stochastic impulsive systems in Hilbert spaces by using Nussbaum's fixed point theorem. In [13], by using stochastic Lyapunov-like approach, sufficient conditions for stochastic $\epsilon$-controllability are formulated. Balachandran et al. [14] researched the controllability of semilinear stochastic integrodifferential systems by using the Picard type iteration. By using the contraction mapping principle, Mahmudov and Zorlu studied the controllability [15] for nonlinear stochastic systems. Moreover, there are some researchers discussing the controllability for the stochastic system driven by fractional Brownian motion; for example, see $[10,16]$.

However, the above authors only consider that $A$ is an infinitesimal generator of a strongly continuous semigroup. But in our work (1), $A(t)$ generates an evolution system $U(t, s)$ on a Hilbert space $H$. If $A(t) \equiv A$ and $B(t) \equiv B$, the works $[7,8]$ are the special cases. We only assume that the linear system is completely controllable. By using the Cauchy-Schwarz inequality, Banach fixed point theorem, and so forth, we prove that the nonlinear system is completely controllable.

The rest of this paper is organized as follows. In the next section, we will introduce some useful preliminaries. In Section 3, some sufficient conditions are established to guarantee the existence and uniqueness of mild solutions of system (1). In Section 4, we will study the completely controllability for nonlinear impulsive stochastic evolution systems. Finally, we present an example to illustrate our main results.

\section{Preliminaries}

Now we introduce some basic definitions, preliminaries, and notations which are used throughout this paper.

Let $(\Omega, \Gamma, P)$ be a complete probability space with probability measure $P$ on $\Omega$. $L_{2}\left(\Omega, \Gamma_{b}, X\right)$ denotes the Hilbert space of all $\Gamma_{b}$-measurable square integrable random variables with values in $X . L_{2}^{\Gamma}(J, X)$ is the Hilbert space of all square integrable and $\Gamma_{t}$-measurable processes with values in $X$. $C\left(J, L_{2}\left(\Omega, \Gamma_{b}, X\right)\right)$ denotes the Banach space of continuous maps from $J$ into $L_{2}\left(\Omega, \Gamma_{b}, X\right)$ satisfying $\sup _{t \in J} E\|x(t)\|^{2}<\infty$.

In order to define the solution of problem (1), we introduce the space $P C\left(J, L_{2}\left(\Omega, \Gamma_{b}, X\right)\right)$ formed by all $\Gamma_{t}$-adapted, $X$-valued processes $\{x(t): t \in J\}$ such that $x$ is continuous at $t \neq t_{k}$ and $x\left(t_{k}^{-}\right)$and $x\left(t_{k}^{+}\right)$exist with $x\left(t_{k}^{-}\right)=x\left(t_{k}\right), k=$ $1,2, \ldots, p$.
In this paper, we assume that $P C\left(J, L_{2}(\Omega, \Gamma, X)\right)$ is endowed with the norm

$$
\|x\|_{P C}=\left(\sup _{t \in[0, b]} E\|x(t)\|^{2}\right)^{1 / 2} .
$$

Then, $\left(P C\left(J, L_{2}(\Omega, \Gamma, X)\right),\|\cdot\|_{P C}\right)$ is a Banach space (see [17]).

We also introduce some basic definitions on fractional Brownian motion ( $\mathrm{fBm})$.

Let $\left(\Omega, \Gamma,\left(\Gamma_{t}, t \in[0, b]\right), P\right)$ be a complete probability space with a filtration satisfying the standard conditions.

Definition 1. The fractional Brownian motion ( $\mathrm{fBm}$ ) with Hurst index $H \in(0,1)$ is a Gaussian process $B_{t}^{H}=\left\{B_{t}^{H}, \Gamma_{t}, t \in\right.$ $[0, b]\}$, having the properties $B_{0}^{H}=0, \mathbb{E} B_{t}^{H}=0$, and $\mathbb{E} B_{t}^{H} B_{s}^{H}=$ $(1 / 2)\left(s^{2 H}+t^{2 H}-|t-s|^{2 H}\right)$.

Let $b>0$, for a linear space $\Upsilon$; there exists $R$-valued step function $\phi \in \Upsilon$ on $[0, b]$, such that

$$
\phi(t)=\sum_{i=1}^{n-1} z_{i} \chi_{\left(t_{i}, t_{i+1}\right]}(t),
$$

where $t \in[0, b], x_{i} \in R$ and $0=t_{1}<t_{2}<\cdots<t_{n}=b$. For $\phi \in \Upsilon$, the Wiener integral with respect to $B^{H}$ can be defined as

$$
\int_{0}^{b} \phi(s) d B^{H}(s)=\sum_{i=1}^{n-1} z_{i}\left(B^{H}\left(t_{i+1}\right)-B^{H}\left(t_{i}\right)\right) .
$$

Let $\mathscr{H}$ be a Hilbert space, which is defined as the closure of $\Upsilon$ with respect to the scalar product $\left\langle\chi_{[0, b]}, \chi_{(0, s]}\right\rangle_{\mathscr{H}}=R_{H}(t, s)$. Then the mapping

$$
\phi=\sum_{i=1}^{n-1} z_{i} \chi_{\left(t_{i}, t_{i+1}\right]} \longmapsto \int_{0}^{b} \phi(s) d B^{H}(s)
$$

is an isometry between $\Upsilon$ and the linear space span $\left\{B^{H}(t)\right.$ : $t \in[0, b]\}$, which can be extended to an isometry between $\mathscr{H}$ and the first Wiener chaos of the fBm $\overline{\operatorname{span}}^{L^{2}(\Omega)}\left\{B^{H}(t)\right.$ : $t \in[0, b]\}$ (see [18]). The image of an element $\phi \in \mathscr{H}$ by this isometry is called the Wiener integral of $\phi$ with respect to $B^{H}$.

Next we give an explicit expression of this integral.

Let us consider the Kernel

$$
K_{H}(t, s)=c_{H} s^{1 / 2-H} \int_{s}^{t}(z-s)^{H-3 / 2} z^{1 / 2-H} d z,
$$

where $c_{H}=(H(2 H-1) / B(2-2 H, H-1 / 2))^{1 / 2}(B(\cdot, \cdot)$ denote the Beta function) and $t>s$. It is easily shown that

$$
\frac{\partial K_{H}(t, s)}{\partial t}=c_{H}\left(\frac{t}{s}\right)^{H-1 / 2}(t-s)^{H-3 / 2}
$$

Let $\mathscr{K}_{H}: \Upsilon \rightarrow L^{2}([0, b])$ be the linear operator, which is defined as

$$
\mathscr{K}_{H} \phi(s)=\int_{s}^{t} \phi(t) \frac{\partial K_{H}}{\partial t}(t, s) d t .
$$


Then $\left(\mathscr{K}_{H} \chi_{[0, b]}\right)(s)=K_{H}(t, s) \chi_{[0, b]}(s)$, and $\mathscr{K}_{H}$ is an isometry between $\Upsilon$ and $L^{2}([0, b])$ which can be extended to $\mathscr{H}$.

We denote $L_{\mathscr{H}}^{2}([0, b])=\left\{\phi \in \mathscr{H}: \mathscr{K}_{H} \phi \in L^{2}([0, b])\right\}$, since $H>1 / 2$; then we get

$$
L^{1 / H}([0, b]) \subset L_{\mathscr{H}}^{2}([0, b])
$$

Moreover, the following lemma holds.

Lemma 2 (see [19]). For $\phi \in L^{1 / H}([0, b])$,

$$
\begin{aligned}
& H(2 H-1) \iint_{0}^{b}|\phi(r)||\phi(z)||r-u|^{2 H-2} d r d z \\
& \quad \leq c_{H}\|\phi\|_{L^{1 / H}([0, b])}^{2} .
\end{aligned}
$$

Let $\left(U,|\cdot|_{U},\langle\cdot, \cdot\rangle_{U}\right)$ and $\left(V,|\cdot|_{V},\langle\cdot, \cdot\rangle_{V}\right)$ be separable Hilbert spaces. $L(V, U)$ denotes the space of all bounded linear operator from $V$ to $U$ and $Q \in L(V, V)$ is a nonnegative self-adjoint operator. Denote by $L_{Q}^{0}(V, U)$ the space of all $\xi \in L(V, U)$ such that $\xi Q^{1 / 2}$ is a Hilbert-Schmidt operator; the norm is given by

$$
|\xi|_{L_{\mathrm{Q}}^{0}(V, U)}^{2}=\left|\xi Q^{1 / 2}\right|_{\mathrm{HS}}^{2}=\operatorname{tr}\left(\xi Q \xi^{*}\right)
$$

Then $\xi$ is a $Q$-Hilbert-Schmidt operator from $V$ to $U$.

Let $\left\{B_{n}^{H}(t)\right\}_{n \in N}$ be a sequence of two-side one-dimensional $\mathrm{fBm}$, which is mutually independent on the complete probability space $(\Omega, \Gamma, P),\left\{e_{n}\right\}_{n \in N}$ be a complete orthonormal basis in $V$. One defines the $V$-valued stochastic process $B_{\mathrm{Q}}^{H}(t)$ as

$$
B_{\mathrm{Q}}^{H}(t)=\sum_{n=1}^{\infty} B_{n}^{H}(t) Q^{(1 / 2)} e_{n}, \quad t \geq 0 .
$$

If $Q$ is a nonnegative self-adjoint trace class operator, then $\sum_{n=1}^{\infty} B_{n}^{H}(t) Q^{(1 / 2)} e_{n}, t \geq 0$ converges in the space $V$; that is, it holds that $B_{Q}^{H}(t) \in L^{2}(\Omega, V)$. Then, we can say that $B_{Q}^{H}(t)$ is a $V$-valued $Q$-cylindrical $\mathrm{fBm}$ with covariance operator $Q$.

Definition 3. Let $\psi:[0, b] \rightarrow L_{Q}^{0}(V, U)$ such that

$$
\sum_{n=1}^{\infty}\left\|\mathscr{K}_{H}\left(\psi Q^{1 / 2}\right) e_{n}\right\|_{L^{2}([0, b], U)}<\infty .
$$

Then for $t \geq 0$, its stochastic integral with respect to the $\mathrm{fBm}$ $B_{\mathrm{Q}}^{H}$ is defined as

$$
\begin{gathered}
\int_{0}^{t} \psi(s) d B_{\mathrm{Q}}^{H}(s)=\sum_{n=1}^{\infty} \int_{0}^{t} \psi(s) Q^{1 / 2} e_{n} d B_{n}^{H}(s) \\
=\sum_{n=1}^{\infty} \int_{0}^{t}\left(\mathscr{K}_{H}\left(\psi Q^{1 / 2} e_{n}\right)\right)(s) d B_{\mathrm{Q}}^{H}(s),
\end{gathered}
$$

where $W$ is a Wiener process.
Notice that if

$$
\sum_{n=1}^{\infty}\left\|\psi Q^{1 / 2} e_{n}\right\|_{L^{1 / H}([0, b], U)}<\infty
$$

then in particular (15) holds, which follows immediately from (13).

The following lemma is obtained as a simple application of Lemma 2.

Lemma 4 (see [19]). For any $\psi:[0, b] \rightarrow L_{Q}^{0}(V, U)$ such that $\sum_{n=1}^{\infty}\left\|\psi Q^{1 / 2} e_{n}\right\|_{U}$ is uniformly convergent for $t \in[0, b]$, and for any $p, q \in[0, b]$ with $p>q$,

$$
\begin{aligned}
& E\left\|\int_{q}^{p} \psi(s) d B_{Q}^{H}(s)\right\|_{U}^{2} \\
& \quad \leq c H(2 H-1)(p-q)^{2 H-1} \sum_{n=1}^{\infty} \int_{q}^{p}\left\|\psi Q^{1 / 2} e_{n}\right\|_{U}^{2} d s .
\end{aligned}
$$

Then

$$
\begin{aligned}
& E\left\|\int_{q}^{p} \psi(s) d B_{Q}^{H}(s)\right\|_{U}^{2} \\
& \quad \leq c H(2 H-1)(p-q)^{2 H-1} \int_{q}^{p}\|\psi(s)\|_{L_{Q}^{0}(V, U)}^{2} d s,
\end{aligned}
$$

where $c=c(H)$.

In the following, let us give some basic properties of the operator $A(t)$.

Let $\{A(t): t \in J\}$ be a family of linear operators and satisfy the following:

$\left(A_{1}\right)$ The domain $D(A(t))=D$ of $A(t)$ is dense in $H$ and independent of $t$, and $A(t)$ is a closed linear operator.

$\left(A_{2}\right)$ For each $t \in J$, the resolvent $R(\lambda, A(t))$ exists for all $\lambda$ with $\operatorname{Re} \lambda \leq 0$ and there is a constant $M>0$ such that $\|R(\lambda, A(t))\| \leq M /(|\lambda|+1)$.

$\left(A_{3}\right)$ For $t, s, \tau \in J$, there exist constants $H>$ and $0<\alpha \leq$ 1 such that

$$
\left\|(A(t)-A(s)) A^{-1}(\tau)\right\| \leq H|t-s|^{\alpha} .
$$

To establish the framework for our main controllability results, we will introduce the following definitions.

Definition 5 (see [4]). A two-parameter family of bounded linear operators $U(t, s), 0 \leq s \leq t \leq b$ on $H$ is called an evolution system if the following two conditions are satisfied:

(i) $U(t, t)=I, U(t, r) U(r, s)=U(t, s)$ for $0 \leq s \leq r \leq t \leq$ $b$.

(ii) $(t, s) \rightarrow U(t, s)$ is strongly continuous for $0 \leq s \leq t \leq$ $b$. 
Definition 6. $X$-valued process $x(t)$ is called a mild solution of (1), if $x(0)=x_{0}, x(t) \in P C\left(J, L_{2}(\Omega, \Gamma, X)\right)$; for each $0 \leq t<$ $b$ and $s \in[0, t)$, the following integral equation holds:

$$
\begin{aligned}
x(t)= & U(t, 0) x_{0} \\
& +\int_{0}^{t} U(t, s)[B(s) u(s)+f(s, x(s), u(s))] d s \\
& +\sum_{0<t_{k}<t} U\left(t, t_{k}\right) I_{k}\left(x\left(t_{k}\right)\right) \\
& +\int_{0}^{t} U(t, s) \sigma(s) d B_{Q}^{H}(s) .
\end{aligned}
$$

The following lemmas are of great importance in the proof of our main result.

Lemma 7 (see [4], Theorem 6.1 in Chapter 5). Under the assumptions $\left(A_{1}\right)-\left(A_{3}\right)$, there is a unique evolution system $U(t, s)$ on $0 \leq s \leq t \leq b$, satisfying the following:

(i) $\|U(t, s)\| \leq C$ for $0 \leq s \leq t \leq b$.

(ii) For $0 \leq s \leq t \leq b, U(t, s): H \rightarrow D$ and $t \rightarrow$ $U(t, s)$ is strongly differentiable in $H$. The derivative $(\partial / \partial t) U(t, s) \in L(H)$ and it is strongly continuous on $0 \leq s \leq t \leq b$. Moreover,

$$
\begin{aligned}
& \frac{\partial}{\partial t} U(t, s)+A(t) U(t, s)=0 \text { for } 0 \leq s \leq t \leq b, \\
& \left\|\frac{\partial}{\partial t} U(t, s)\right\|=\|A(t) U(t, s)\| \leq \frac{C}{t-s},
\end{aligned}
$$

$\left\|A(t) U(t, s) A^{-1}(s)\right\| \leq C \quad$ for $0 \leq s \leq t \leq b$.

(iii) For every $v \in D(A(t)), t \in J, U(t, s) v$ is differentiable with respect to $s$ on $0 \leq s \leq t \leq b$, and

$$
\frac{\partial}{\partial s} U(t, s) v=U(t, s) A(s) v .
$$

\section{Existence Result}

In this section, we will give the existence results for system (1). We will assume the following conditions:

$\left(H_{1}\right)$ There exist constants $L_{1}, L_{2}>0$ such that

$$
\begin{aligned}
& \left\|f\left(t, x_{1}, u_{1}\right)-f\left(t, x_{2}, u_{2}\right)\right\|^{2} \\
& \quad \leq L_{1}\left\|x_{1}-x_{2}\right\|^{2}+L_{2}\left\|u_{1}-u_{2}\right\|^{2}
\end{aligned}
$$

for a.e. $t \in J$, for all $x_{1}, x_{2} \in H, u_{1}, u_{2} \in U$.

$\left(H_{2}\right)$ There exists a constant $L_{3}>0$ such that

$$
\|f(t, x, u)\|^{2} \leq L_{3}\left(1+\|x\|^{2}\right)
$$

for all $x \in H$ and a.e. $t \in J$.

$\left(H_{3}\right)$ Function $\sigma:[0,+\infty) \rightarrow L_{2}^{0}(X, Y)$ satisfies $\int_{0}^{b}\|\sigma(s)\|^{2} d s \leq \infty$.
$\left(H_{4}\right)$ There exist constants $c_{k}, d_{k}>0(k=1,2, \ldots, p)$ such that, for each $x, y \in H$,

$$
\begin{aligned}
\left\|I_{k}(x)-I_{k}(y)\right\|^{2} & \leq c_{k}\|x-y\|^{2}, \\
\left\|I_{k}(x)\right\|^{2} & \leq d_{k}\left(1+\|x\|^{2}\right) .
\end{aligned}
$$

Now, let us consider the existence result for system (1).

Theorem 8. Assume that hypotheses $\left(H_{1}\right)-\left(H_{4}\right)$ hold. Then for any $u \in L_{2}(J, U)$ the impulsive stochastic system (1) has a unique mild solution in $L_{2}(\Omega, \Gamma, X)$ provided that

$$
C^{2}\left(L_{1} b^{2}+p \sum_{0<t_{k}<t} c_{k}\right)<1 .
$$

Proof. Define an operator $F: X_{2} \rightarrow X_{2}$ by

$$
\begin{aligned}
(F x)(t)= & U(t, 0) x_{0}+\int_{0}^{t} U(t, s) f(s, x(s), u(s)) d s \\
& +\int_{0}^{t} U(t, s) B(s) u(s) d s \\
& +\sum_{0<t_{k}<t} U\left(t, t_{k}\right) I_{k}\left(x\left(t_{k}\right)\right) \\
& +\int_{0}^{t} U(t, s) \sigma(s) d B_{Q}^{H}(s) .
\end{aligned}
$$

By using the Banach contraction mapping principle, we will show that the operator $F$ has a unique fixed point. To prove that, we divide the subsequent proof into two steps:

Step 1. For any $x \in L_{2}(\Omega, \Gamma, X)$, let us show that $t \rightarrow F(x)(t)$ is continuous on $J$ in the $L_{2}(\Omega, \Gamma, X)$-sense.

Let $0<t<t+\delta<b$, here $t, t+\delta \in J \backslash\left\{t_{1}, t_{2}, \ldots, t_{m}\right\}$, and $\delta>0$ be sufficiently small. Then we obtain

$$
\begin{aligned}
& E\|(F x)(t+\delta)-(F x)(t)\|^{2} \leq 8 E \| U(t+\delta, 0) x_{0} \\
& -U(t, 0) x_{0}\left\|^{2}+8 E\right\| \int_{0}^{t}[U(t+\delta, s)-U(t, s)] \\
& \cdot f(s, x(s), u(s)) d s\left\|^{2}+8 E\right\| \int_{t}^{t+\delta} U(t+\delta, s) \\
& \cdot f(s, x(s), u(s)) d s\left\|^{2}+8 E\right\| \int_{0}^{t}[U(t+\delta, s) \\
& -U(t, s)] B(s) u(s) d s\left\|^{2}+8 E\right\| \int_{t}^{t+\delta} U(t+\delta, s) \\
& \cdot B(s) u(s) d s \|^{2}
\end{aligned}
$$




$$
\begin{aligned}
& +8 E \| \sum_{0<t_{k}<t}\left[U\left(t+\delta, t_{k}\right) I_{k}\left(x\left(t_{k}\right)\right)\right. \\
& \left.-U\left(t, t_{k}\right) I_{k}\left(x\left(t_{k}\right)\right)\right]\left\|^{2}+8 E\right\| \int_{0}^{t}[U(t+\delta, s) \\
& -U(t, s)] \sigma(s) d B_{Q}^{H}(s) \|^{2} \\
& +8 E\left\|\int_{t}^{t+\delta} U(t, s) \sigma(s) d B_{Q}^{H}(s)\right\|^{2} \\
& \leq 8\left\{\|U(t+\delta, 0)-U(t, 0)\|^{2} E\left\|x_{0}\right\|^{2}\right. \\
& +t L_{3} \int_{0}^{t}\|U(t+\delta, s)-U(t, s)\|^{2} \\
& \cdot \sup _{s \in J} E\left(1+\|x(s)\|^{2}\right) d s \\
& +C^{2} \delta \int_{t}^{t+\delta} \sup _{s \in J} E\left(1+\|x(s)\|^{2}\right) d s \\
& +t \int_{0}^{t}\|U(t+\delta, s)-U(t, s)\|^{2} E\|B(s) u(s)\|^{2} d s \\
& +C^{2} \delta \int_{t}^{t+\delta} E\|B(s) u(s)\|^{2} d s \\
& +p \sum_{0<t_{k}<t}\left\|U\left(t+\delta, t_{k}\right)-U\left(t, t_{k}\right)\right\|^{2} E\left\|I_{k}\left(x\left(t_{k}\right)\right)\right\|^{2} \\
& +c H(2 H-1) t^{2 H-1} \int_{0}^{t}\|U(t+\delta, s)-U(t, s)\|^{2} \\
& \cdot\|\sigma(s)\|_{U}^{2} d s+c C^{2} H(2 H-1) \\
& \left.\cdot t^{2 H-1} \int_{t}^{t+\delta}\|\sigma(s)\|_{U}^{2} d s\right\} \text {. }
\end{aligned}
$$

Then, by the strong continuous of $U(t, s)$ and the Lebesgue's dominated convergence theorem, we know that the right hand of (27) tends to 0 as $\delta \rightarrow 0$. Hence, $F(x)(t)$ is continuous on $J$ in the $L_{2}(\Omega, \Gamma, X)$-sense.

Step 2. We prove that $F$ is a contraction mapping. Let $x, y \in$ $P C\left(J, L_{2}(\Omega, U)\right)$ be two mild solutions of (1); then

$$
\begin{gathered}
E\|(F x)(t)-(F y)(t)\|_{H}^{2} \leq E \| \int_{0}^{t} U(t, s) \\
\cdot[f(s, x(s), u(s))-f(s, y(s), u(s))] d s \\
+\sum_{0<t_{k}<t} U\left(t, t_{k}\right)\left[I_{k}\left(x\left(t_{k}\right)\right)-I_{k}\left(y\left(t_{k}\right)\right)\right] \|^{2}
\end{gathered}
$$

$$
\begin{aligned}
& \leq 2 t E \int_{0}^{t}\|U(t, s)\|^{2} \| f(s, x(s), u(s)) \\
& -f(s, y(s), u(s))\left\|_{H}^{2} d s+2 p \sum_{0<t_{k}<t}\right\| U\left(t, t_{k}\right) \|^{2} \\
& \cdot E\left\|I_{k}\left(x\left(t_{k}\right)\right)-I_{k}\left(y\left(t_{k}\right)\right)\right\|_{H}^{2} \leq 2 t C^{2}\left(L_{1} b\right. \\
& \left.+p \sum_{0<t_{k}<t} c_{k}\right) \sup _{t \in J} E\|x(t)-y(t)\|_{H}^{2} .
\end{aligned}
$$

Inequality (28) equates to

$$
\begin{aligned}
& \sup _{t \in J} E\|(F x)(t)-(F y)(t)\|_{H}^{2} \\
& \quad \leq C^{2}\left(L_{1} b^{2}+p \sum_{0<t_{k}<t} c_{k}\right) \sup _{t \in J} E\|x(t)-y(t)\|_{H}^{2} .
\end{aligned}
$$

Since $C^{2}\left(L_{1} b^{2}+p \sum_{0<t_{k}<t} c_{k}\right)<1$, we know that $F$ is a contraction mapping. Hence a unique fixed point $x(\cdot)$ in $P C\left(J, L_{2}(\Omega, U)\right)$ exists, which is the mild solution of problem (1).

\section{Controllability Result}

In this section, we discuss the controllability results for system (1). Before starting, we consider the following assumption:

$\left(H_{5}\right)$ The linear operator $L_{0}^{b} \in \mathscr{L}\left(U, L_{2}\left(\Omega, \Gamma_{b}, X\right)\right)$ is defined by

$$
L_{0}^{b} u=\int_{0}^{b} U(b, s) B(s) u(s) d s
$$

and has an inverse operator $\left(L_{0}^{b}\right)^{-1}$ which takes values in $L_{2}(J, U) \backslash \operatorname{ker} L_{0}^{b}$, where $\operatorname{ker} L_{0}^{b}=\left\{x \in(J, U), L_{0}^{b} x=0\right\}$, and there are positive constants $M_{b}, M_{L}$ such that $\|B\|^{2} \leq$ $M_{b},\left\|\left(L_{0}^{b}\right)^{-1}\right\|^{2} \leq M_{L}$.

To the readers' convenience, we give the definitions of controllability as follows.

Definition 9. System (1) is said to be completely controllable on the interval $J$ if

$$
\mathscr{R}_{t}\left(x_{0}\right)=L_{2}\left(\Omega, \Gamma_{b}, X\right)
$$

that is, all the points in $L_{2}\left(\Omega, \Gamma_{b}, X\right)$ can be exactly reached from arbitrary initial condition $h$ and $x_{0}$ at time $b$. 
Theorem 10. Assume that hypotheses $\left(H_{1}\right)-\left(H_{5}\right)$ hold. Then the impulsive stochastic system (1) is completely controllable on $J$, if

$$
\begin{aligned}
& 3\left(b C^{2} L_{1} b+b C^{2} p \sum_{0<t_{k}<t} d_{k}\right. \\
& \left.+\frac{2 C^{6} M_{b}^{2} M_{L} b\left(b^{2} L_{1}+p \sum_{k=1}^{p} d_{k}\right)}{1-b L_{2}}\right) \leq 1 .
\end{aligned}
$$

Proof. Fix $b>0$ and let $Q_{b}=P C\left(J, L_{2}(\Omega, \Gamma, X)\right)$ be the Banach space of all functions from $J$ into $L_{2}(\Omega, \Gamma, X)$, endowed with the supremum norm

$$
\|\mu\|_{Q_{b}}=\left(\sup _{t \in[0, b]} E\|\mu(t)\|^{2}\right)^{1 / 2} .
$$

Let us consider the set

$$
G_{b}=\left\{x \in Q_{b}: x(0)=x_{0}\right\} .
$$

We easily know that $G_{b}$ is a closed subset of $Q_{b}$ equipped with norm $\|\cdot\|_{Q_{b}}$.

By condition $\left(H_{5}\right)$, we choose the feedback control function as

$$
\begin{aligned}
& u_{x}(t)=B^{*}(t) U^{*}(b, t) E\left\{( L _ { 0 } ^ { b } ) ^ { - 1 } \left(x_{b}-U(b, 0) x_{0}\right.\right. \\
& -\int_{0}^{b} U(b, s) f\left(s, x(s), u_{x}(s)\right) d s \\
& -\sum_{k=1}^{p} U\left(b, t_{k}\right) I_{k}\left(x\left(t_{k}\right)\right) \\
& \left.\left.-\int_{0}^{b} U(t, s) \sigma(s) d B_{Q}^{H}(s) \mid \Gamma_{t}\right)\right\} .
\end{aligned}
$$

The operator $\Psi$ defined on $\|\cdot\|_{Q_{b}}$ by

$$
\begin{aligned}
& \Psi(x)(t)=U(t, 0) x_{0}+\int_{0}^{t} U(t, s) B(s) B^{*}(s) U^{*}(b, s) \\
& \cdot E\left[( L _ { 0 } ^ { b } ) ^ { - 1 } \left(x_{b}-U(b, 0) x_{0}\right.\right. \\
& -\int_{0}^{b} U(b, \eta) f(s, x(\eta), u(\eta)) d \eta \\
& -\sum_{k=1}^{p} U\left(b, t_{k}\right) I_{k}\left(x\left(t_{k}\right)\right) \\
& \left.\left.-\int_{0}^{b} U(b, \eta) \sigma(\eta) d B_{Q}^{H}(\eta) \mid \Gamma_{t}\right)\right] d s+\int_{0}^{t} U(t, \\
& s) f(s, x(s), u(s)) d s+\sum_{0<t_{k}<t} U\left(t, t_{k}\right) I_{k}\left(x\left(t_{k}\right)\right) \\
& +\int_{0}^{t} U(t, s) \sigma(s) d B_{Q}^{H}(s) .
\end{aligned}
$$

has a fixed point on $J$.
To prove that, we divide the subsequent proof into two steps.

Step 1. For any $x \in G_{b}$, let us show that $t \rightarrow \Psi(x)(t)$ is continuous on $J$ in the $L_{2}(\Omega, \Gamma, X)$-sense.

Let $0<t<t+\delta<b$, here $t, t+\delta \in J \backslash\left\{t_{1}, t_{2}, \ldots, t_{m}\right\}$, and $\delta>0$ be sufficiently small. Then we obtain

$$
\begin{aligned}
& E\|\Psi(x)(t+\delta)-\Psi(x)(t)\|^{2} \leq 8 E \| U(t+\delta, 0) x_{0} \\
& -U(t, 0) x_{0}\left\|^{2}+8 E\right\| \int_{0}^{t}[U(t+\delta, s)-U(t, s)] \\
& \cdot f(s, x(s), u(s)) d s\left\|^{2}+8 E\right\| \int_{t}^{t+\delta} U(t+\delta, s) \\
& \cdot f(s, x(s), u(s)) d s\left\|^{2}+8 E\right\| \int_{0}^{t}[U(t+\delta, s) \\
& -U(t, s)] B(s) B^{*}(s) U^{*}(b, s)\left(L_{0}^{b}\right)^{-1}\left(x_{b}\right. \\
& -U(b, 0) x_{0}-\int_{0}^{b} U(b, \eta) f(s, x(\eta), u(\eta)) d \eta \\
& -\sum_{k=1}^{p} U\left(b, t_{k}\right) I_{k}\left(x\left(t_{k}\right)\right) \\
& \left.-\int_{0}^{b} U(b, \eta) \sigma(\eta) d B_{\mathrm{Q}}^{H}(\eta)\right) d s \|^{2} \\
& +8 E \| \int_{t}^{t+\delta} U(t+\delta, s) B(s) B^{*}(s) U^{*}(b, s)\left(L_{0}^{b}\right)^{-1} \\
& \cdot\left(x_{b}-U(b, 0) x_{0}\right. \\
& -\int_{0}^{b} U(b, \eta) f(s, x(\eta), u(\eta)) d \eta \\
& -\sum_{k=1}^{p} U\left(b, t_{k}\right) I_{k}\left(x\left(t_{k}\right)\right) \\
& \left.-\int_{0}^{b} U(b, \eta) \sigma(\eta) d B_{\mathrm{Q}}^{H}(\eta)\right) d s \|^{2} \\
& +8 E \| \sum_{0<t_{k}<t}\left[U\left(t+\delta, t_{k}\right) I_{k}\left(x\left(t_{k}\right)\right)\right. \\
& \left.-U\left(t, t_{k}\right) I_{k}\left(x\left(t_{k}\right)\right)\right]\left\|^{2}+8 E\right\| \int_{0}^{t}[U(t+\delta, s) \\
& -U(t, s)] \sigma(s) d B_{\mathrm{Q}}^{H}(s) \|^{2} \\
& +8 E\left\|\int_{t}^{t+\delta} U(t, s) \sigma(s) d B_{\mathrm{Q}}^{H}(s)\right\|^{2}
\end{aligned}
$$




$$
\begin{aligned}
& \leq 8\left\{\|U(t+\delta, 0)-U(t, 0)\|^{2} E\left\|x_{0}\right\|^{2}\right. \\
& +t L_{3} \int_{0}^{t}\|U(t+\delta, s)-U(t, s)\|^{2} \\
& +\sup _{s \in J} E\left(1+\|x(s)\|^{2}\right) d s \\
& +C^{2} \delta \int_{t}^{t+\delta} \sup _{s \in J} E\left(1+\|x(s)\|^{2}\right) d s \\
& +5 t M_{b}^{2} C^{2} M_{L}\left[\left\|x_{b}\right\|+C^{2}\left\|x_{0}\right\|+b C^{2}\right. \\
& +\sup _{\eta \in J}\left(1+\|x(\eta)\|^{2}\right)+p C^{2} \sum_{k=0}^{p} d_{k} \sup _{\eta \in J}\left(1+\|x(\eta)\|^{2}\right) \\
& +c H(2 H-1) t^{2 H-1} \int_{0}^{t}\|U(t+\delta, s)-U(t, s)\|^{2} \\
& \left.+c C^{2}(2 H-1) b^{2 H-1} \int_{0}^{b}\|\sigma(\eta)\|^{2} d \eta\right] \\
& +\sum_{\eta \in J}{ }^{t}\left\|U\left(t+\delta, t_{k}\right)-U\left(t, t_{k}\right)\right\|^{2} E\left\|I_{k}\left(x\left(t_{k}\right)\right)\right\|^{2} \\
& +\int_{0}\left\|M_{b}^{2}(t+\delta, s)-U(t, s)\right\|^{2} d s \\
& +M_{L}\left[\left\|x_{b}\right\|+C^{2}\left\|x_{0}\right\|+b C^{2}\right.
\end{aligned}
$$

$$
\begin{aligned}
& \cdot\|\sigma(s)\|_{U}^{2} d s+c C^{2} H(2 H-1) \\
& \left.\cdot t^{2 H-1} \int_{t}^{t+\delta}\|\sigma(s)\|_{U}^{2} d s\right\} .
\end{aligned}
$$

Then, by the strong continuous of $U(t, s)$ and the Lebesgue's dominated convergence theorem, we know that the right hand of (27) tends to 0 as $\delta \rightarrow 0$. Hence, $F(x)(t)$ is continuous on $J$ in the $L_{2}(\Omega, \Gamma, X)$-sense.

Step 2. We prove that $\Psi$ is a contraction mapping.

Let $x, y \in P C\left(J, L_{2}(\Omega, U)\right)$ be two mild solutions of (1); then

$$
\begin{aligned}
& E\|\Psi(x)(t)-\Psi(y)(t)\|_{H}^{2} \leq 3 E \| \int_{0}^{t} U(t, s) \\
& \cdot\left[f\left(s, x(s), u_{x}(s)\right)-f\left(s, y(s), u_{y}(s)\right)\right] d s \|^{2} \\
& +3 E\left\|\int_{0}^{t} U(t, s) B(s)\left[u_{x}(s)-u_{y}(s)\right] d s\right\|^{2} \\
& +3 E\left\|\sum_{0<t_{k}<t} U\left(t, t_{k}\right)\left[I_{k}\left(x\left(t_{k}\right)\right)-I_{k}\left(y\left(t_{k}\right)\right)\right]\right\|^{2} \\
& \quad \leq 3 J_{1}+3 J_{2}+3 J_{3} .
\end{aligned}
$$

We can easily show that

$$
\begin{aligned}
& J_{1} \leq t C^{2} L_{1} b \sup _{t \in J} E\|x(t)-y(t)\|_{H}^{2}, \\
& J_{3} \leq t C^{2} p \sum_{0<t_{k}<t} d_{k} \sup _{t \in J} E\|x(t)-y(t)\|_{H}^{2} .
\end{aligned}
$$

Since

$$
\begin{aligned}
& E\left\|u_{x}(t)-u_{y}(t)\right\|^{2} \leq E \| B^{*}(t) U^{*}(b, t)\left(L_{0}^{b}\right)^{-1} \\
& \quad\left\{\int_{0}^{b} U(b, s)\left(f\left(s, x(s), u_{x}(s)\right)-f\left(s, y(s), u_{y}(s)\right)\right) d s-\sum_{k=1}^{p} U\left(b, t_{k}\right)\left(I_{k}\left(x\left(t_{k}\right)\right)-I_{k}\left(y\left(t_{k}\right)\right)\right)\right\} \|^{2} \\
& \quad \leq 2 C^{4} M_{b} M_{L}\left(E\left\|\int_{0}^{b}\left[f\left(s, x(s), u_{x}(s)\right)-f\left(s, y(s), u_{y}(s)\right)\right] d s\right\|^{2}+E\left\|\sum_{k=1}^{p}\left(I_{k}\left(x\left(t_{k}\right)\right)-I_{k}\left(y\left(t_{k}\right)\right)\right)\right\|^{2}\right) \\
& \quad \leq 2 C^{4} M_{b} M_{L}\left(b \int_{0}^{b} E\left(L_{1}\|x(s)-y(s)\|^{2}+L_{2}\left\|u_{x}(s)-u_{y}(s)\right\|^{2}\right) d s+p \sum_{k=1}^{p} d_{k}\|x-y\|_{P C}^{2}\right) \leq 2 C^{4} M_{b} M_{L}\left(b^{2} L_{1}\right. \\
& \left.\quad+p \sum_{k=1}^{p} d_{k}\right)\|x-y\|_{P C}^{2}+b L_{2} \int_{0}^{b} E\left\|u_{x}(s)-u_{y}(s)\right\|^{2} d s,
\end{aligned}
$$


we have

$$
\begin{aligned}
& \sup _{t \in J} E\left\|u_{x}(t)-u_{y}(t)\right\|^{2} \\
& \quad \leq \frac{2 C^{4} M_{b} M_{L}\left(b^{2} L_{1}+p \sum_{k=1}^{p} d_{k}\right)}{1-b L_{2}}\|x-y\|_{P C}^{2} .
\end{aligned}
$$

Hence

$$
J_{2} \leq \frac{2 C^{6} M_{b}^{2} M_{L} b\left(b^{2} L_{1}+p \sum_{k=1}^{p} d_{k}\right)}{1-b L_{2}}\|x-y\|_{P C}^{2} .
$$

From above inequalities, we obtain

$$
\begin{aligned}
& E\|\Psi(x)(t)-\Psi(y)(t)\|^{2} \leq 3\left(b C^{2} L_{1} b\right. \\
& +b C^{2} p \sum_{0<t_{k}<t} d_{k} \\
& \left.+\frac{2 C^{6} M_{b}^{2} M_{L} b\left(b^{2} L_{1}+p \sum_{k=1}^{p} d_{k}\right)}{1-b L_{2}}\right)\|x-y\|_{P C}^{2} .
\end{aligned}
$$

Since

$$
\begin{aligned}
& 3\left(b C^{2} L_{1} b+b C^{2} p \sum_{0<t_{k}<t} d_{k}\right. \\
& \left.+\frac{2 C^{6} M_{b}^{2} M_{L} b\left(b^{2} L_{1}+p \sum_{k=1}^{p} d_{k}\right)}{1-b L_{2}}\right)<1,
\end{aligned}
$$

therefore $\Psi$ is a contraction mapping. A unique fixed point $x(\cdot)$ exists in $P C\left(J, L_{2}(\Omega, \Gamma, X)\right)$, which is the mild solution of problem (1). Problem (1) is completely controllable on $J$.

\section{An Example}

Consider the following semilinear stochastic differential equations of the following form:

$$
\begin{aligned}
\partial z(\theta, t)= & -\sum_{i=1}^{n} \frac{\partial}{\partial \theta_{i}}\left(p(\theta, t) \frac{\partial z(\theta, t)}{\partial \theta_{i}}\right) \partial t \\
& +f(\theta, t, z(\theta, t), u(\theta, t)) \partial t \\
& +\sigma(t) \partial B_{Q}^{H}(t), \quad \text { in }[0, \tau] \times \Omega, \\
z\left(\theta, t_{k}^{+}\right)= & x\left(\theta, t_{k}^{-}\right)+I_{k}\left(x\left(\theta, t_{k}\right)\right), \\
z(\theta, t)= & 0 \quad \text { on } \partial \Omega \times[0, \tau], \\
z(\theta, 0)= & z_{0}(\theta) \quad \theta \in \Omega,
\end{aligned}
$$

where $\Omega$ is a bounded open set in $R^{n}, f: \Omega \times J \times$ $R^{n} \times R^{m} \rightarrow R, g: \Omega \times J \times R^{n} \times R^{m} \rightarrow L(R)$ are nonlinear functions, measurable with respect to $\theta$ and almost everywhere continuous with respect to $t$, and continuous in the last two variables.
We define the function $p: J \times \Omega \rightarrow R$ as follows:

(i) $p(\theta, t) \geq c>0,(\theta, t) \in \Omega \times J$.

(ii) $p$ is Lipschitz continuous with respect to $t$ and continuous differentiable in $\theta$, and $p(\cdot, t) \in L_{\infty}$.

Let $H=L_{2}(\Omega)$ and $D=H^{2} \cap H_{0}^{2}(\Omega)$; then $D$ is dense in $H$. By [20], the operator $A(t): D \subset H \rightarrow H, t \in[0, \tau]$, defined by

$$
\langle A(t) z, v\rangle=\sum_{0}^{n} \int_{\Omega}-p(y, t)\left(\frac{\partial z}{\partial y_{i}}\right)\left(\frac{\partial v}{\partial y_{i}}\right) d y,
$$

$$
z, v \in D \text {. }
$$

Then, system (45) can be rewritten as

$$
\begin{aligned}
d z(t)= & {[A(t) z(t)+f(t, z(t), u(t))] d t } \\
& +\sigma(t) d B_{\mathrm{Q}}^{H}(t), \quad t \in[0, \tau] \\
z(0)= & z_{0} \in H .
\end{aligned}
$$

For the operator $A$, we can obtain that there exist two constants $b_{1} \geq 0, b_{2}>0$, such that

$$
\begin{gathered}
\langle A(t) x, x\rangle=\sum_{0}^{n} \int_{\Omega}-p(y, t)\left|\frac{\partial x}{\partial y_{i}}\right|^{2} d y \geq b_{1}, \\
x \in D, \\
\|A(t) x-A(s) x\| \leq b_{2}|t-s|\|x\|, \quad x \in D .
\end{gathered}
$$

It is shown that $\left(A_{1}\right)-\left(A_{3}\right)$ are satisfied. Therefore, if we impose suitable conditions on $f$ and $d B_{Q}^{H}(t)$ to ensure $\left(H_{1}\right)-\left(H_{5}\right)$ are satisfied, system (45) will be completely controllable by Theorem 10 .

\section{Conflict of Interests}

The authors declare that there is no conflict of interests regarding the publication of this paper.

\section{Acknowledgments}

The research is supported by Guangxi Science and Technology Research Project for Universities no. LX2014105, no. LX2014106, and no. ZD2014044, Guangxi Universities for Nationalities Youth Project no. 2013MDQN043, the Chinese NSF no. 61402121, no. 11371003, and no. 11461006, Guangxi NSF no. 2013GXNSFAA019342, no. 2011GXNSFA018154, and no. 2012GXNSFGA060003, the Bagui scholarship project of Guangxi, the Science Research Project 2014 of the ChinaASEAN Study Center (Guangxi Science Experiment Center) of Guangxi University for Nationalities, the Innovation Project of Guangxi Graduate Education (gxunYCSZ2014121), the Science and Technology Foundation of Guangxi under Grant no. 10169-1, and the Scientific Research Project no. 201012MS274 from Guangxi Education Department. 


\section{References}

[1] D. Bainov and P. Simeonov, Impulsiv Differential Equations: Peridic Solutions and Applictiona, Longman, Essex, UK, 1993.

[2] V. Lakshmikantham, D. D. Bainov, and P. S. Simeonov, Theory of Impulsive Differential Equations, World Scientific, Singapore, 1989.

[3] Y. V. Rogovchenko, "Nonlinear impulse evolution systems and applications to population models," Journal of Mathematical Analysis and Applications, vol. 207, no. 2, pp. 300-315, 1997.

[4] A. Pazy, Semigroups of Linear Operators and Applications to Partial Differential Equations, Springer, New York, NY, USA, 1983.

[5] M. Besalú and C. Rovira, "Stochastic delay equations with nonnegativity constraints driven by fractional Brownian motion," Bernoulli, vol. 18, no. 1, pp. 24-45, 2012.

[6] J. Unterberger, "Stochastic calculus for fractional Brownian motion with Hurst exponent $H>1 / 4$ : a rough path method by analytic extension," The Annals of Probability, vol. 37, no. 2, pp. 565-614, 2009.

[7] D. T. Nguyen, "Mackey-Glass equation driven by fractional Brownian motion," Physica A: Statistical Mechanics and Its Applications, vol. 391, no. 22, pp. 5465-5472, 2012.

[8] B. Boufoussi and S. Hajji, "Neutral stochastic functional differential equations driven by a fractional Brownian motion in a Hilbert space," Statistics \& Probability Letters, vol. 82, no. 8, pp. 1549-1558, 2012.

[9] T. Caraballo, M. J. Garrido-Atienza, and T. Taniguchi, "The existence and exponential behavior of solutions to stochastic delay evolution equations with a fractional Brownian motion," Nonlinear Analysis. Theory, Methods \& Applications, vol. 74, no. 11, pp. 3671-3684, 2011.

[10] H. M. Ahmed, "Controllability of impulsive neutral stochastic differential equations with fractional Brownian motion," IMA Journal of Mathematical Control and Information, 2014.

[11] R. E. Kalman, "Controllability of linear dynamical systems," Contributions to the Theory of Differential Equations, vol. 1, pp. 190-213, 1963.

[12] R. Subalakshmi and K. Balachandran, "Approximate controllability of nonlinear stochastic impulsive integrodifferential systems in Hilbert spaces," Chaos, Solitons \& Fractals, vol. 42, no. 4, pp. 2035-2046, 2009.

[13] J. Klamka and L. Socha, "Some remarks about stochastic controllability," IEEE Transactions on Automatic Control, vol. 22, no. 5, pp. 880-881, 1977.

[14] K. Balachandran, S. Karthikeyan, and J.-H. Kim, "Controllability of semilinear stochastic integrodifferential systems," Kybernetika, vol. 43, no. 1, pp. 31-44, 2007.

[15] N. I. Mahmudov and S. Zorlu, "Controllability of non-linear stochastic systems," International Journal of Control, vol. 76, no. 2, pp. 95-104, 2003.

[16] H. M. Ahmed, "Approximate controllability of impulsive neutral stochastic differential equations with fractional Brownian motion," Advances in Difference Equations, vol. 113, pp. 1-11, 2014.

[17] Z. M. Yan and X. X. Yan, "Existence of solutions for a impulsive nonlocal stochastic functional integrodifferential inclusion in Hilbert spaces," Zeitschrift für angewandte Mathematik und Physik, vol. 64, no. 3, pp. 573-590, 2013.

[18] S. Tindel, C. A. Tudor, and F. Viens, "Stochastic evolution equations with fractional Brownian motion," Probability Theory and Related Fields, vol. 127, no. 2, pp. 186-204, 2003.
[19] Y. Mishura, Stochastic Calculus for Fractional Brownian Motion and Related Processes, Springer, Berlin, Germany, 2008.

[20] W. M. Bian, "Controllability of nonlinear evolution systems with preassigned responses," Journal of Optimization Theory and Applications, vol. 100, no. 2, pp. 265-285, 1999. 


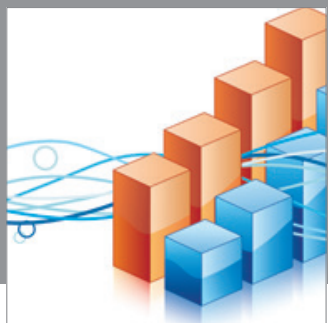

Advances in

Operations Research

mansans

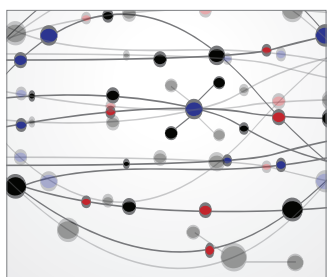

The Scientific World Journal
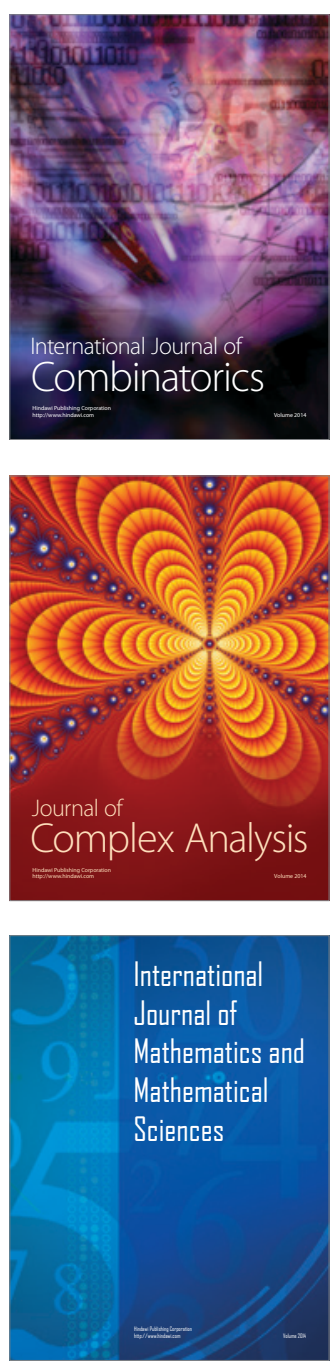
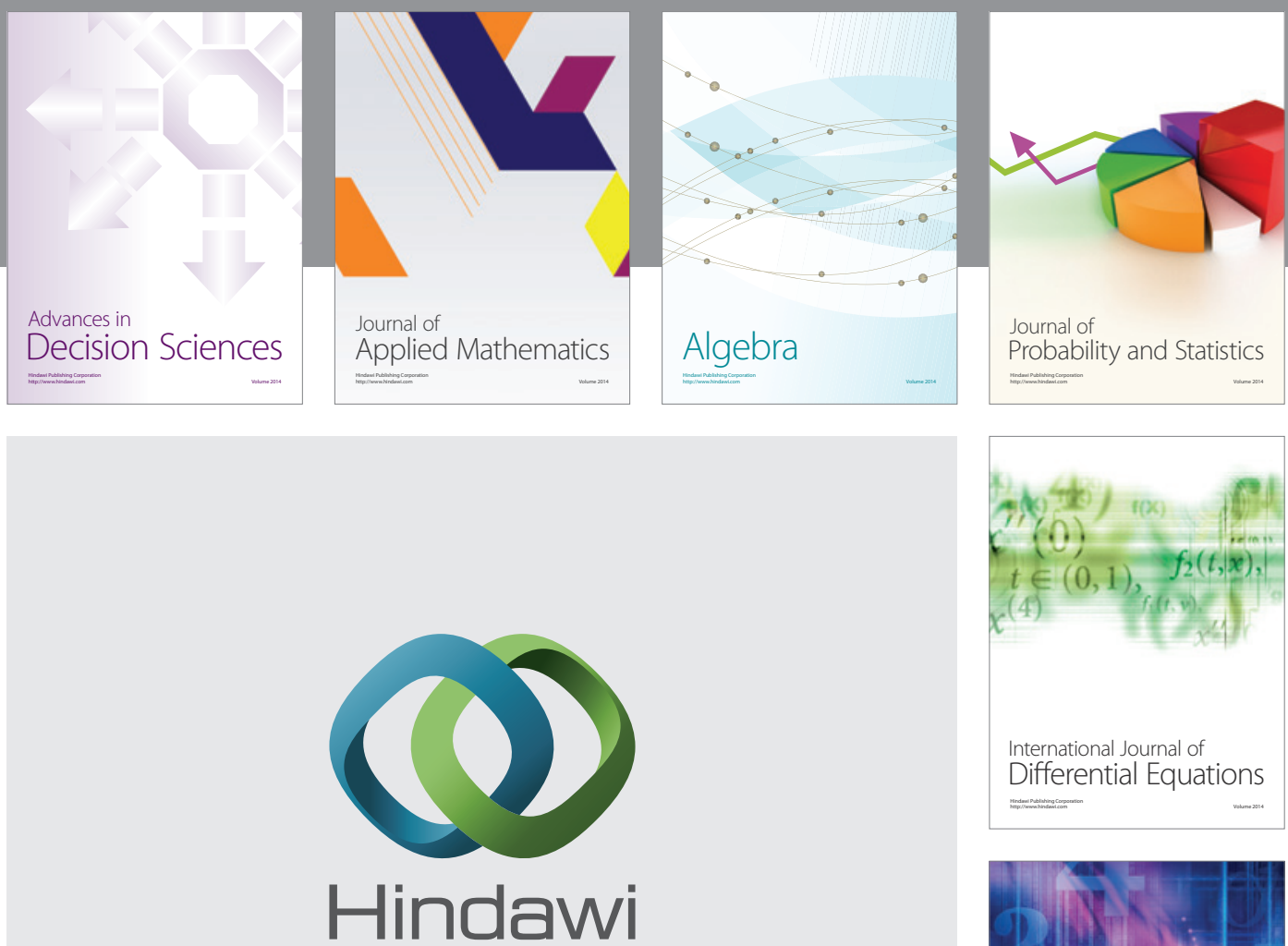

Submit your manuscripts at http://www.hindawi.com
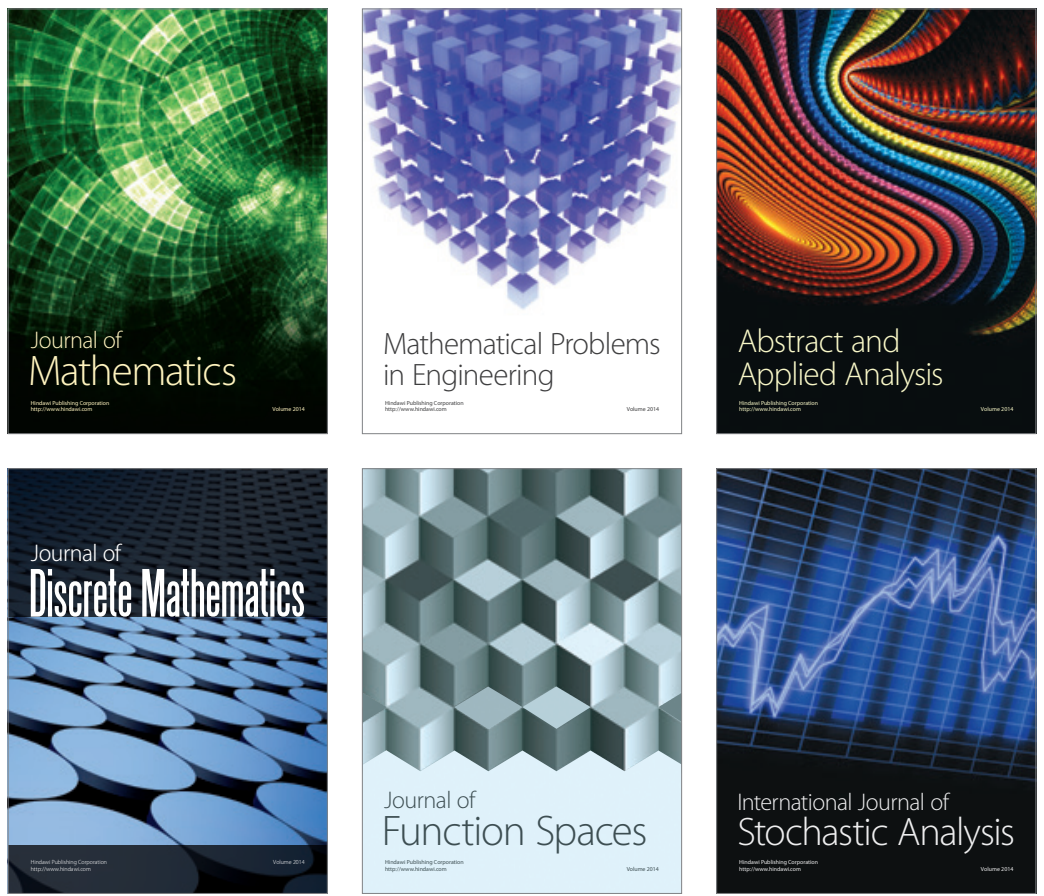

Journal of

Function Spaces

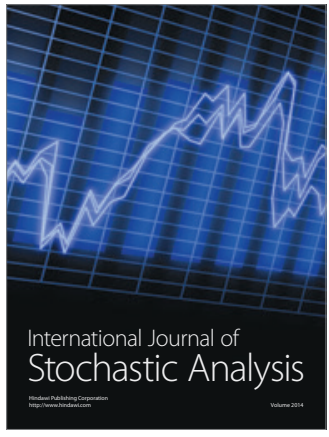

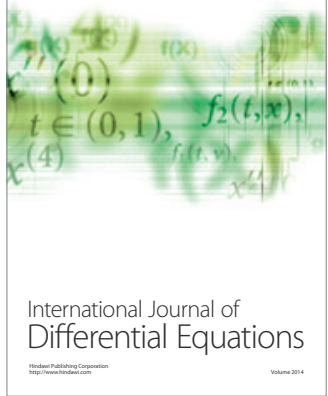
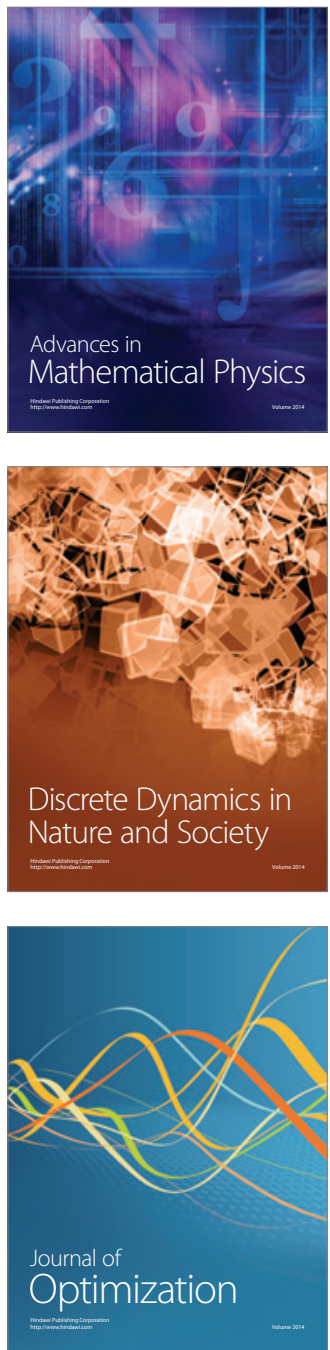